\title{
Contribution à l'étude de la relation entre les différentes formes du phosphore dans les sédiments d'une retenue de barrage eutrophe en climat méditerranéen (El Kansera, Maroc) Contribution to the study of the relationship between different species of phosphorus in an eutrophic reservoir sediments in mediterranean climate (El Kansera, Morocco)
}

\author{
A. Abdallaoui, M. Derraz, M. Z. Bhenabdallah et S. Lek
}

Volume 11, numéro 1, 1998

URI : https://id.erudit.org/iderudit/705299ar

DOI : https://doi.org/10.7202/705299ar

Aller au sommaire du numéro

\section{Éditeur(s)}

Université du Québec - INRS-Eau, Terre et Environnement (INRS-ETE)

ISSN

0992-7158 (imprimé)

1718-8598 (numérique)

Découvrir la revue

Citer cet article

Abdallaoui, A., Derraz, M., Bhenabdallah, M. Z. \& Lek, S. (1998). Contribution à l'étude de la relation entre les différentes formes du phosphore dans les sédiments d'une retenue de barrage eutrophe en climat méditerranéen (El Kansera, Maroc). Revue des sciences de l'eau / Journal of Water Science, 11(1), 101-116. https://doi.org/10.7202/705299ar

\begin{abstract}
Résumé de l'article
Le présent travail porte sur l'évaluation des différentes formes de phosphore, dans les sédiments d'un réservoir eutrophe en climat méditerranéen (El Kansera, Maroc) et la détermination des relations entre ces formes. L'application d'un schéma de fractionnement chimique du phosphore a permis de déterminer les proportions de quatre formes différentes : le phosphore lié au fer $\mathrm{Fe}(\mathrm{OOH})-\mathrm{P}$, le phosphore lié au calcium CaCO3-P, le phosphore organique soluble dans l'acide POSA et le phosphore organique résiduel POR. La forme biodisponible a été déterminée par ailleurs, par des bioessais en utilisant une Chlorophycée (Scenedesmus crassus) isolée à partir des eaux de la retenue du barrage étudié.

Une étude par analyse factorielle des correspondances a mis en évidence l'influence de la nature des sédiments étudiés et de leurs propriétés physico-chimiques sur la répartition des formes du phosphore dans les sédiments. En période de basses eaux, les sédiments sont caractérisés par la prédominance des formes inorganiques ; alors qu'en période de hautes eaux, la forme POSA est la plus abondante. La confrontation des résultats obtenus par la méthode des bioessais avec ceux relatifs aux extractions chimiques séquentielles de phosphore a montré par ailleurs, que la forme biodisponible du phosphore est fortement corrélée aux formes inorganiques $\{\mathrm{Fe}(\mathrm{OOH})-\mathrm{P}$ et CaCO3-P\} et indépendante vis à vis des formes organiques $\{\mathrm{POSA}$ et POR\}.
\end{abstract}




\title{
Contribution à l'étude de la relation entre les différentes formes du phosphore dans les sédiments d'une retenue de barrage eutrophe en climat méditerranéen (EI Kansera, Maroc)
}

\author{
Contribution to the study of the relationship among \\ different forms of phosphorus in the sediments \\ of a eutrophic reservoir in a Mediterranean climate \\ (El Kansera, Morocco)
}

\section{A. ABDALLAOUI ${ }^{*}$, M. DERRAZ ${ }^{2}$, M.Z. BHENABDALLAH ${ }^{1}$ et S. LEK ${ }^{3}$}

Reçu le 9 septembre 1996, accepté le 30 juillet $1997^{\star}$.

We have studied in this work the different forms of phosphorus in the sediments of a eutrophic reservoir in a Mediterranean climate (EI Kansera, Morocco). The studied sediments have been collected at eight sampling stations located at different points in the middle of the lake during two sampling sessions in contrasting weather conditions: the first sampling occurred in November 1992, a period of autumn mixing and low water levels, whereas the second took place in January 1994 during a high water level period, just after the first winter floods of the Beht river whose waters are retained in the studied reservoir.

The evaluation of the different forms of phosphorus was carried out using both a chemical extraction method and a bioassay method. The application of the chosen chemical extraction scheme allowed the determination of the proportions of four different phosphorus forms: iron-bound $\{\mathrm{Fe}(\mathrm{OOH})-\mathrm{P}\}$, calciumbound $\left\{\mathrm{CaCO}_{3}-\mathrm{P}\right\}$, acid-soluble organic $\{\mathrm{POSA}\}$ and residual organic $\{\mathrm{POR}\}$. The bioavailable form \{Pbio\}, representing less than $32 \%$ of the total sediment phosphorus content, has been evaluated using a Scenedesmus crassus algal strain isolated from El Kansera reservoir waters.

According to the results of our analyses, the concentrations of iron-bound phosphorus are higher overall than those of calcium-bound phosphorus. The sediments collected during the low water period contain spatially stable values of POSA (0.03-0.09 $\left.\mathrm{mg} \cdot \mathrm{g}^{-1}\right)$. These values are lower than those determined in the sediments collected during the high water period $\left(0.24-0.42 \mathrm{mg} \cdot \mathrm{g}^{-1}\right)$. This form represents less than $11 \%$ of the total phosphorus in the low water period whereas it represents more than $25 \%$ and up to $56 \%$ of the total phosphorus in the filling period. This result can be explained by the freshness of the organic

1. Département de Chimie, Faculté des Sciences, Béni M'Hamed, BP 4010, Meknès, Maroc.

2. Département de Biologie, Faculté des Sciences, Béni M'Hamed, BP 4010, Meknès, Maroc.

3. CNRS-UMR 5576 - CESAC, Bat. 4R3, Université Paul Sabatier, 118, route de Narbonne, 31062 Toulouse, Cedex, France.

* Les commentaires seront reçus jusqu'au 6 novembre 1998. 
matter supplied by the continuous water flow in the high water period and the degradation of this organic matter during the low water period. Moreover, the anaerobic conditions of the hypolimnetic layers and the low external supply during the low water period favour organic phosphorus mineralisation.

A Factorial Correspondence analysis study has shown the influence of the nature and the physico-chemical properties of the studied sediments on the distribution of the different phosphorus forms. In the low level period, the predominance of the inorganic forms is obvious while POSA is the most abundant form in the period of rising water levels. This differentiation can be explained by the different transport dynamics of suspended organic or inorganic particles according to the water level and also to the external or internal supply of the organic matter.

We have also determined the statistical relations among the estimated different forms of phosphorus in El Kansera reservoir sediments. This statistical analysis was carried out to establish the correlation between the bioavailable phosphorus determined by the bioassay method and the phosphorus forms determined by chemical speciation. The goal of the study was to determine, according to statistically significant correlations, the chemical forms of the bioavailable phosphorus contained in the studied sediments.

The comparison between the results we have obtained by the bioassay method and those obtained by sequential chemical extraction has shown that the bioavailable form of phosphorus is strongly correlated with the inorganic forms $\{\mathrm{Fe}(\mathrm{OOH})-\mathrm{P}\}$ and $\left\{\mathrm{CaCO}_{3}+\mathrm{P}\right\}$. The high correlation found between the ironbound form of phosphorus and the bioavailable one is in good agreement with previous work described in the literature that has shown that an important part of the iron-bound and aluminium-bound phosphorus is bioavailable, whatever the extraction method may be.

It has also been demonstrated that the forms $\{\mathrm{Pbio}\},\{\mathrm{Fe}(\mathrm{OOH})-\mathrm{P}\},\left\{\mathrm{CaCO}_{3}-\mathrm{P}\right\}$ and $\{$ Pinorg\}are notably independent from the $\{P O S A\}$ and $\{$ Porg\} forms, the correlation coefficients being relatively low. The residual organic form of phosphorus \{POR\} does not seem to be linked to any variable. Therefore, we can say that this form is not bioavailable for the Scenedesmus crassus species.

Key-words : phosphorus, bioavailable phosphorus, chemical extractions, bioassays. sediments, eutrophic reservoirs.

Le présent travail porte sur l'évaluation des différentes formes de phosphore, dans les sédiments d'un réservoir eutrophe en climat méditerranéen (El Kansera, Maroc) et la détermination des relations entre ces formes. L'application d'un schéma de fractionnement chimique du phosphore a permis de déterminer les proportions de quatre formes différentes : le phosphore lié au fer $\mathrm{Fe}(\mathrm{OOH})-\mathrm{P}$, le phosphore lié au calcium $\mathrm{CaCO}_{3}-\mathrm{P}$, le phosphore organique soluble dans l'acide POSA et le phosphore organique résiduel POR. La forme biodisponible a été déterminée par ailleurs, par des bioessais en utilisant une Chlorophycée (Scenedesmus crassus) isolée à partir des eaux de la retenue du barrage étudié.

Une étude par analyse factorielle des correspondances a mis en évidence l'influence de la nature des sédiments étudiés et de leurs propriétés physicochimiques sur la répartition des formes du phosphore dans les sédiments. En période de basses eaux, les sédiments sont caractérisés par la prédominance des formes inorganiques ; alors qu'en période de hautes eaux, la forme POSA est la plus abondante. La confrontation des résultats obtenus par la méthode des bioessais avec ceux relatifs aux extractions chimiques séquentielles de 
phosphore a montré par ailleurs, que la forme biodisponible du phosphore est fortement corrélée aux formes inorganiques $\left\{\mathrm{Fe}(\mathrm{OOH})-\mathrm{P}\right.$ et $\left.\mathrm{CaCO}_{3}-\mathrm{P}\right\}$ et indépendante vis à vis des formes organiques \{POSA et POR\}.

Mots clés : phosphore, phosphore biodisponible, extractions chimiques, bioessais, sédiments, retenues de barrages eutrophes.

\section{1 - INTRODUCTION}

L'eutrophisation des retenues de barrages est un processus qui fait appel à des mécanismes biologiques et physico-chimiques complexes. Au cours de ce dernier siècle, ce processus naturel s'est considérablement accéléré et amplifié sous l'influence humaine. En effet, par son activité industrielle, agricole et domestique, l'homme rejette dans son environnement aquatique des quantités importantes d'éléments fertilisants, qui viennent perturber les cycles biogéochimiques naturels. Ces apports sont responsables du niveau global du phénomène de l'eutrophisation des plans d'eau. Parmi ces éléments fertilisants, le phosphore joue un rôle important dans ce phénomène, car il constitue un facteur stimulant particulièrement la croissance des algues (DILLON et RIGLER, 1974 ; VOLLENWEIDER, 1976 ; RECKHOW et CHAPRA, 1983 ; LAFFORGUE, 1990).

Par ailleurs, une importante part du phosphore déversé dans les écosystèmes aquatiques se retrouve finalement plus ou moins fortement associée aux sédiments du fond de ces écosystèmes. Or, il se peut que dans certaines conditions et par divers mécanismes, ce phosphore soit relargué par ces sédiments dans la colonne d'eau. D'un autre côté, il a été établi que seule une partie du phosphore stocké dans les sédiments est biodisponible (GROBLER et DAVIES, $1979 ; 1981$ ) et donc susceptible de favoriser la prolifération algale qui entraîne une détérioration de la qualité de l'eau et pose notamment de sérieux problèmes lors de son traitement de potabilisation.

L'importance du rôle joué par le phosphore dans le phénomène de l'eutrophisation des lacs et des réservoirs dépend donc, non seulement de la quantité globale du phosphore stockée dans ses sédiments, mais aussi et essentiellement de la proportion des formes biodisponibles liées à ces sédiments.

La détermination des teneurs des différentes formes du phosphore dans les sédiments des retenues de barrage est donc d'une grande importance dans l'étude de leur eutrophisation et constitue plus généralement, une contribution appréciable à la compréhension du fonctionnement de ces milieux.

Dans les pays méditerranéens à climat semi-aride comme le Maroc, le phénomène de l'eutrophisation des plans d'eau se pose avec une grande acuité (LAHLOU, 1987). Il semble donc utile de chercher à déterminer les diverses formes du phosphore liées aux sédiments et d'évaluer en particulier les teneurs en formes biodisponibles.

Cette spéciation du phosphore dans les sédiments peut être effectuée par de nombreuses méthodes qui sont malheureusement toutes critiquables. Ces méthodes peuvent être regroupées en deux grands types:

- Les méthodes de bioessais qui sont basées sur l'utilisation des sédiments comme source unique de phosphore pour la croissance d'une culture monoalgale 
(WILLIAMS et al., 1980 ; BOSTROM et al., 1988). Cependant, la méthodologie et les problèmes théoriques associés à ces biotests nécessitent une attention particulière (GOLTERMAN, 1988). Compte tenu de la grande variabilité de la composition des sédiments et des mécanismes qui peuvent provoquer le relargage, aucune méthode de bioessais n'a pu être acceptée de façon universelle et les auteurs utilisent des protocoles expérimentaux très différents, ce qui rend plus difficiles les comparaisons des résultats obtenus (DORICH et al., 1980; GOLTERMAN, 1988).

- Les méthodes de fractionnement chimique qui sont basées sur des extractions séquentielles des formes du phosphore (HIELTJES et LIJKLEMA, 1980 ; PSENNER et PUCSKO, 1988 ; BONZONGO et al., 1989 ; OLILA et al., 1995). Ces méthodes sont en général aussi critiquées, car elles emploient des extractants trop agressifs et insuffisamment spécifiques (DE GROOT, 1992). Ces extractants conduisent parfois à de mauvaises interprétations des phénomènes liés aux échanges à l'interface eau-sédiment (BONZONGO et al., 1989), car ils supposent que ces compartiments chimiques ont des disponibilités biologiques déterminées.

Pour notre part, nous avons cherché dans le présent travail à déterminer les relations statistiques entre les différentes formes de phosphore présentes dans les sédiments d'une retenue de barrage eutrophe située au centre du Maroc: El Kansera. Cette détermination a été faite afin d'établir notamment la corrélation entre le phosphore biodisponible évalué par la méthode des bioessais et les formes de phosphore déterminées par spéciation chimique. Le principal objectif d'une telle étude étant de déterminer, à travers l'établissement de corrélations statistiquement significatives, les formes chimiques du phosphore biodisponible dans les sédiments du milieu.

Afin d'atteindre cet objectif, nous avons commencé par évaluer, dans les sédiments étudiés, les teneurs en phosphore total, les proportions des différentes formes de phosphore déterminées par une méthode des extractions chimiques et les teneurs en phosphore biodisponible évaluées par des bioessais.

Ce travail s'inscrit dans le cadre d'un large programme d'investigations pluridisciplinaires, visant l'étude de l'eutrophisation de la retenue susmentionnée et la détermination d'approches de solutions permettant, à plus ou moins long terme, de réduire l'intensité de ce phénomène et ses répercussions négatives.

\section{2 - MATÉRIEL ET MÉTHODES}

\subsection{Milieu d'étude}

La retenue de barrage El Kansera, située au nord-ouest du Maroc $\left(34^{\circ} 00^{\prime} \mathrm{N}\right.$, $-5^{\circ} 55^{\prime} \mathrm{W}$ ), se trouve sur l'oued Beht (fig. 1), affluent de la rive gauche de l'oued Sebou. Elle présente une hydrologie très contrastée avec une capacité de $266 \cdot 10^{6} \mathrm{~m}^{3}$, correspondant à une superficie de $18 \mathrm{~km}^{2}$ au niveau normal (122,5 N.G.M.). Sa profondeur moyenne est de $16,5 \mathrm{~m}$ et le point le plus profond est de $28 \mathrm{~m}$. Le temps de séjour moyen de l'eau dans la retenue est d'environ 2 à 36 mois (DERRAZ, 1995).

L'importance de ce barrage réside dans le fait qu'il contribue à l'approvisionnement en eau potable de deux villes (Khémisset et Tiflet) et à l'irrigation de plus 

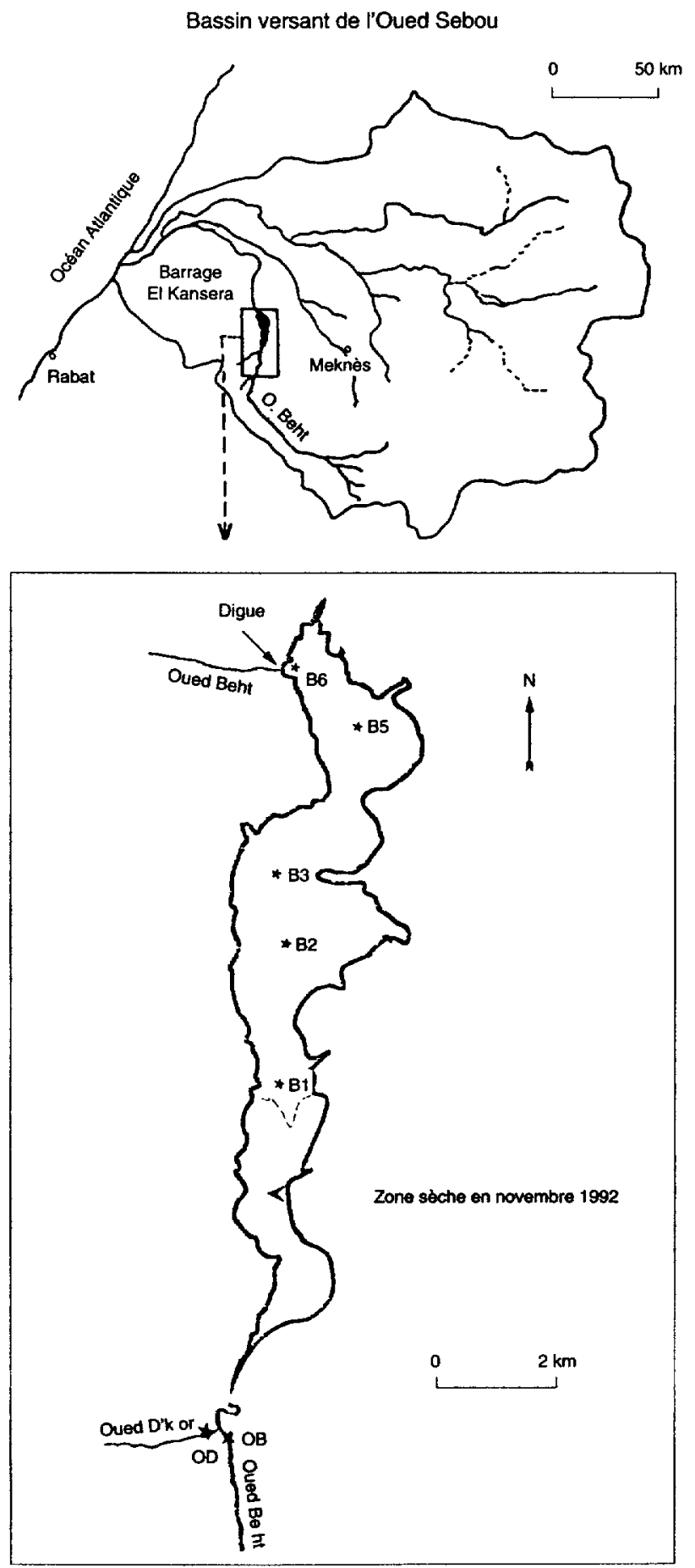

Figure 1 Situation de la retenue du barrage El Kansera avec les stations de prélèvement El Kansera Reservoir with location of sampling stations. 
de 30000 ha de terres cultivables tout en apportant à l'électricité marocaine un appoint annuel d'environ 13 millions de Kwh.

Le bassin versant de l'oued Beht sur lequel a été érigé le barrage El Kansera, d'une superficie de $4540 \mathrm{~km}^{2}$ et de couverture végétale réduite, est constitué de collines marneuses du Miocène, traversées par une vallée orientée vers le nord.

Les particularités hydromorphométriques de cette retenue en font un écosystème lacustre particulièrement adapté à l'étude du fonctionnement des réservoirs à fond plat, sous climat méditerranéen chaud (DERRAZ, 1995).

En effet, la gestion hydraulique et les caractéristiques hydromorphologiques de la retenue de barrage El Kansera ont pour conséquence un marnage important lié à la fluctuation du volume des eaux emmagasinées dans ce réservoir. La forte dénivellation du plan d'eau en fin d'été et en début d'automne de chaque année coïncide avec la formation sur la surface du plan d'eau de blooms d'algues plus ou moins importants. Cette prolifération algale est probablement liée, entre autres, à la biodisponibilité du phosphore dans le milieu. Cette biodisponibilité étant elle même dépendante des échanges de phosphore à l'interface eau-sédiments, lesquels sont généralement influencés par les caractéristiques hydromorphologiques de la retenue.

L'étude de la répartition des formes de phosphore dans les sédiments de cette retenue peut donc contribuer positivement à la compréhension des mécanismes régissant le processus d'apparition des blooms de cyanobactéries. Ce qui pourrait nous amener à définir des approches de solutions, permettant à terme de réduire l'eutrophisation de la retenue considérée.

Les échantillons de sédiments superficiels ont été prélevés au niveau de huit stations (fig. 1), à l'aide d'une benne de type Ekman, lors de deux campagnes effectuées dans des conditions climatiques contrastées : la première en novembre 1992, période de mélange automnal et de basses eaux, et la deuxième en janvier 1994, après l'arrivée dans la retenue des premières crues hivernales, correspondant à une période de hautes eaux.

Le choix des huit stations de prélèvement a été fait de manière à tenir compte dans l'étude statistique de l'influence des deux facteurs suivants:

- la contribution des apports externes en phosphore drainés par l'oued Beht qui alimente la retenue (station OB), et par son affluent l'oued D'kor (station OD) dans lequel sont déversées les eaux usées de la ville de Khémisset ;"

- les caractéristiques hydromorphologiques de la retenue, telles que la profondeur ou la répartition en sous-bassins, qui sont suffisamment variables pour nous amener à choisir six stations (B1 à B6), situées sur le grand axe du lac (lit de la retenue).

Le fait d'avoir effectué deux campagnes de prélèvement d'échantillons de sédiments se justifie par la volonté de tenir compte dans le traitement statistique du facteur temps, qui a une influence majeure, selon les périodes (basses et hautes eaux), sur l'homogénéité des sédiments, leur composition ou leurs origines (sédiments anciens ou correspondant à une déposition récente de matières en suspension amenées par les crues).

\subsection{Bioessais}

En raison de son rythme de multiplication rapide et de son mode de culture simple, Scenedesmus crassus (Chlorophycée) a été adoptée pour les bioessais. 
Une souche de cette espèce a été isolée des eaux prélevées près du rivage de la retenue du barrage. Une détermination des constantes spécifiques de croissance et de photosynthèse de cette souche a été effectuée (DERRAZ et al., 1995).

Les cultures ont été réalisées en milieu artificiel non renouvelé (batch-culture) avec un apport d'air permettant de limiter l'appauvrissement du milieu en dioxyde de carbone. Ce mode de culture simple est une technique employée dans la majorité des bioessais puisqu'elle permet d'évaluer la biomasse maximale d'algue produite (HANNA et DAUTA, 1983).

Les expériences ont été conduites dans des erlenmyers en verre borosilicaté d'une capacité de $500 \mathrm{ml}$. Dans chaque flacon, $200 \mathrm{ml}$ de culture sont ajoutés à environ $1,2 \cdot 10^{6}$ cellules $\cdot \mathrm{ml}^{-1}$ et $50 \mathrm{ml}$ d'une suspension homogène, contenant $0,1 \mathrm{~g}$ de sédiment humide et préalablement tamisé à $50 \mu \mathrm{m}$. L'inoculum utilisé provient d'une pré-culture carencée en phosphore. Parallèlement, une gamme d'étalonnage à été réalisée dans les mêmes conditions à des concentrations croissantes en phosphore apporté sous forme de $\mathrm{K}_{2} \mathrm{HPO}_{4}$.

L'ensemble est soumis aux conditions de culture suivantes : éclairement continu avec une intensité lumineuse de $130 \mu \mathrm{E} \cdot \mathrm{m}^{-2} \cdot \mathrm{s}^{-1}$ apportée par une lampe phytoclaude de $400 \mathrm{~W}$, sous une température moyenne de $30^{\circ} \mathrm{C}$ (DERRAZ, 1995 ; DERRAZ et al., 1995).

La croissance des cultures a été suivie par la mesure de la densité optique à l'aide d'un spectrophotomètre (Varian) à $750 \mathrm{~nm}$ dans des cuves de $5 \mathrm{~cm}$ de trajet optique. Les prélèvements ont été effectués, également pour les comptages de la densité cellulaire (sur cellule de Malassez) et pour le dosage de la chlorophylle a (extraction à l'éthanol).

La quantité de phosphore biodisponible dans les échantillons de sédiments a été estimée par comparaison de la biomasse algale maximale produite dans les bioessais à celle obtenue à partir de cultures-étalons.

\subsection{Fractionnement chimique}

Sur la figure 2, nous avons présenté le schéma utilisé pour le fractionnement chimique du phosphore des sédiments. Cette technique est inspirée des travaux de GOLTERMAN et BOOMAN (1988), de DE GROOT et GOLTERMAN (1990) et de GOLTERMAN et DE GROOT (1994).

La première étape s'effectue en traitant $1 \mathrm{~g}$ de sédiment humide préalablement débarrassé des débris supérieurs à $2 \mathrm{~mm}$, avec une solution de Ca-NTA-dithionite $\left(0,022 \mathrm{M} \mathrm{CaCO}_{3}, 0,02 \mathrm{M} \mathrm{NTA}, 0,1 \mathrm{M}\right.$ TRIS (2-amino-2(hydroxyméthyl)-1,3-propanediol) et $1 \mathrm{~g}$ de $\mathrm{Na}_{2} \mathrm{~S}_{2} \mathrm{O}_{4} / 100 \mathrm{ml}$ ) à $\mathrm{pH}=8$, pour extraire le phosphore lié au fer sous forme de $\mathrm{Fe}(\mathrm{OOH})$-P. Le dosage du phosphore ainsi solubilisé dans le surnageant, par les méthodes décrites ci-dessous, a montré que cette étape nécessite plus de quatre opérations consécutives pour extraire la totalité de cette forme.

La deuxième étape s'effectue en traitant le résidu de la première étape avec une solution de $\mathrm{Na}$-EDTA $(0,05 \mathrm{M})$ à $\mathrm{pH}=8$. Le sédiment étant déjà débarrassé du fer actif, lors de la première étape, l'EDTA forme un complexe avec le calcium du sédiment, ainsi la forme $\mathrm{CaCO}_{3}$-P se trouve en solution. Pour extraire la totalité de cette forme 2 à 3 extractions ont été nécessaires lors de cette étape.

La troisième étape s'effectue en une seule extraction en milieu acide $\left(\mathrm{H}_{2} \mathrm{SO}_{4}\right.$ $0,5 \mathrm{M})$. Selon GOLTERMAN et DE GROOT (1994), cette étape nous permet l'extraction du phosphore organique soluble en milieu acide (POSA). 


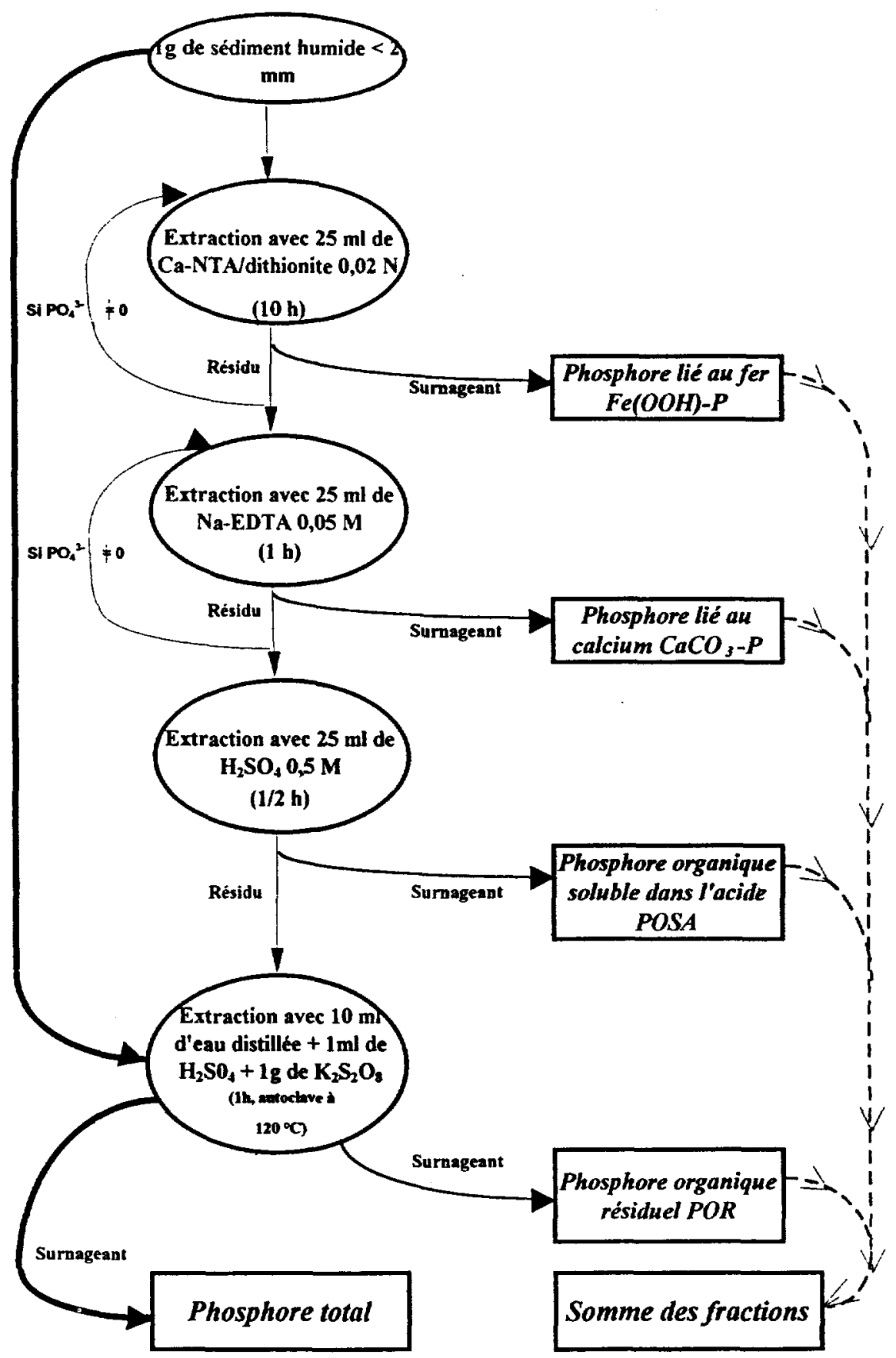

Figure 2 Schéma d'extractions séquentielles du phosphore réalisées sur les sédiments de la retenue du barrage El Kansera.

Phosphorus sequential extraction procedure used to analyse the sediment of El Kansera Reservoir. 
La dernière étape correspond à une minéralisation totale du résidu en présence de $10 \mathrm{ml}$ d'eau distillée, $1 \mathrm{ml}$ de $\mathrm{H}_{2} \mathrm{SO}_{4}$ concentré et $1 \mathrm{~g}$ de $\mathrm{K}_{2} \mathrm{~S}_{2} \mathrm{O}_{8}$. Cette minéralisation s'effectue à $120^{\circ} \mathrm{C}$ à l'autoclave pendant une heure. Elle nous permet la solubilisation du phosphore résiduel qui correspond essentiellement à du phosphore organique (POR).

Pour la solubilisation du phosphore total, nous avons pratiqué cette minéralisation directement sur $1 \mathrm{~g}$ de sédiment (BONZONGO, 1990) humide préalablement débarrassé des débris supérieurs à $2 \mathrm{~mm}$.

La procédure analytique suivie pour l'application de ce schéma est la suivante :

Dans un tube à centrifuger, nous mettons $1 \mathrm{~g}$ de sédiment humide (fraction inférieure à $2 \mathrm{~mm}$ ) et $25 \mathrm{ml}$ d'extractant. Après des temps variables selon l'étape d'extraction de chaque forme, l'ensemble est centrifugé pendant $20 \mathrm{~min}$ à 4000 tours $\cdot \min ^{-1}$, le surnageant est récupéré. Le résidu est remis en suspension dans $25 \mathrm{ml}$ de l'extractant suivant.

Le phosphore sous forme d'orthophosphate est dosé dans le surnageant, par la technique colorimétrique, selon sa concentration, soit par la méthode de MURPHY et RILEY (1962) utilisant le molybdate d'ammonium et l'acide ascorbique comme réactifs de base $(\lambda=700 \mathrm{~nm})$, soit par la méthode décrite par MOTOMIZU et al. (1983) utilisant le vert de malachite comme réactif de base $(\lambda=650 \mathrm{~nm})$. Le spectrophotomètre utilisé est de type WPA modèle S 106.

Pour chaque extraction, une gamme de solutions étalons est préparée dans les mêmes conditions. Les résultats sont exprimés en $\mathrm{mg}$ de phosphore par gramme de sédiment sec.

\subsection{Traitement statistique des données}

Afin de mettre en évidence les relations entre les variables que constituent les fractions de phosphore et les relations entre les périodes d'échantillonnage et les stations de prélèvement, nous avons effectué une analyse factorielle des correspondances (BENZÉCRI, 1973). Cette méthode a été utilisée ici, pour décrire de façon synthétique notre jeu de données, à l'aide du logiciel Statlab ${ }^{\circledR}$ (édité par CNET) version 2.0. Les stations $O B$ et $O D$, qui ne sont pas situées dans la retenue du barrage El Kansera, ont été introduites comme des individus supplémentaires. Le phosphore biodisponible, estimé par les bioessais, a été pris comme variable supplémentaire. II représente en effet, la fraction la plus importante dont il faut prouver la corrélation avec les autres formes du phosphore déterminées par les extractions séquentielles chimiques. Le $\mathrm{P}_{\text {inorg }}$ et le $\mathrm{P}_{\text {org }}$ ont été utilisés aussi comme des variables supplémentaires, étant donné que le premier a été estimé en additionnant $\mathrm{Fe}(\mathrm{OOH})-\mathrm{P}$ et $\mathrm{CaCO}_{3}-\mathrm{P}$ et que le deuxième a été estimé en additionnant le POSA et POR.

\section{3 - RÉSULTATS ET DISCUSSION}

\subsection{Variations des formes de phosphore}

Pour donner une idée sur les résultats analytiques, qui ont servi à l'étude des relations entre les différentes formes de phosphore dans les sédiments étudiés, 
nous allons présenter une brève description des teneurs de ces formes ainsi que de leur variation.

Sur le tableau 1 , sont reportés les paramètres statistiques relatifs aux valeurs obtenues des concentrations des différentes formes du phosphore déterminées par les extractions séquentielles chimiques, ainsi que celles du phosphore biodisponible déterminé par la méthode des bioessais.

Tableau 1 Paramètres statistiques (en $\left.\mathrm{mg} \cdot \mathrm{g}^{-1}\right)$ des variables étudiées $(n=16)$.

SD : écart-type ;

CV : coefficient de variation (en \%).

Table 1 Statistical parameters $\left(m g \cdot g^{-1}\right)$ of the variables used in this study $(n=16)$. SD: standard deviation; CV: coefficient of variation (\%).

\begin{tabular}{|lcccccccc|}
\hline & $\mathbf{P}_{\text {bio }}$ & $\mathbf{F e}(\mathbf{O O H})-\mathbf{P}$ & $\mathbf{C a C O}_{\mathbf{3}}-\mathbf{P}$ & $\mathbf{P}_{\text {inorg }}$ & POSA & POR & P $_{\text {org }}$ & $\mathbf{P}_{\text {tot }}$ \\
\hline Minimum & 0,060 & 0,040 & 0,030 & 0,070 & 0,030 & 0,120 & 0,180 & $\mathbf{0}, 480$ \\
Maximum & 0,300 & 0,340 & 0,110 & 0,450 & 0,420 & 0,250 & 0,600 & $\mathbf{1 , 0 9 0}$ \\
Moyenne & 0,124 & 0,089 & 0,043 & 0,133 & 0,174 & 0,188 & 0,363 & 0,713 \\
SD & 0,061 & 0,069 & 0,020 & 0,088 & 0,122 & 0,035 & 0,119 & 0,178 \\
CV \% & 49,04 & 77,94 & 46,16 & 65,93 & 72,93 & 18,33 & 32,77 & 24,94 \\
\hline
\end{tabular}

\subsubsection{Bioessais}

Les tests des bioessais, réalisés sur l'ensemble des échantillons prélevés dans la retenue du barrage El Kansera, ont montré que les proportions du phosphore lié au sédiment utilisable par les algues $\left(P_{b i o}\right)$ sont variables. Elles oscillent entre 0,06 et $0,30 \mathrm{mg} \cdot \mathrm{g}^{-1}$ (tabl. 1), soit entre 11 et $32 \%$ du phosphore total $\left(P_{\text {tot }}\right)$. Le coefficient de variation est de $49 \%$. La valeur la plus élevée $\left(0,30 \mathrm{mg} \cdot \mathrm{g}^{-1}\right)$ correspond à l'échantillon prélevé dans l'oued D'kor (station OD) en novembre 1992 et ayant la teneur la plus élevée en phosphore total $\left(1,1 \mathrm{mg} \cdot \mathrm{g}^{-1}\right.$ de sédiment sec). Selon WILLIAMS et al. (1980), le pourcentage d'utilisation du phosphore sédimentaire par les algues est d'autant plus élevé que les concentrations en phosphore total sont fortes.

Les sédiments déposés à l'entrée de la retenue en période d'étiage, où les apports de l'oued D'kor sont proportionnellement importants, présentent de forts pourcentages du phosphore biodisponible ( $32 \%$ de $\mathrm{P}_{\text {tot }}$ ) par rapport aux stations relativement plus protondes, situées vers la digue du barrage ( $14 \%$ du $P_{\text {tot }}$ ).

Dans l'oued Beht (station $\mathrm{OB}$ ), les apports de phosphore biodisponible $(0,06$ et $0,08 \mathrm{mg} \cdot \mathrm{g}^{-1}$ ) par l'ensemble des sédiments prélevés, paraissent insuffisants pour engendrer une croissance significative de Scenedesmus crassus.

Par ailleurs, dans la retenue du barrage El Kansera, les valeurs moyennes du phosphore lié au sédiment, biodisponible pour Scenedesmus crassus, sont plus faibles que celles trouvées par Afdali (1993) pour le réservoir Hassan I (au Maroc) $\left(29,6\right.$ à $49,5 \%$ du $\left.P_{\text {tot }}\right)$, beaucoup plus profond et récemment mis en eau.

\subsubsection{Fractionnement chimique}

Les teneurs en phosphore total des sédiments des stations étudiées présentent de faibles variations d'une station à l'autre. En effet, elles varient entre 0,48 et $1,1 \mathrm{mg} \cdot \mathrm{g}^{-1}$ de sédiment $\mathrm{sec}$, avec une moyenne de $0,71 \mathrm{mg} \cdot \mathrm{g}^{-1}$ et un 
coefficient de variation de $24,94 \%$ (tabl. 1). La plus forte valeur a été enregistrée au niveau des sédiments de la station OD, en novembre 92, sur l'oued D'kor. Rappelons que ce cours d'eau véhicule les eaux usées domestiques et industrielles, chargées en polyphosphates (contenus notamment dans les détergents), de la ville de Khémisset. En période d'étiage (novembre 92), une grande partie des orthophosphates des eaux de ce cours, qui sont essentiellement des rejets, se retrouve fixée par les sédiments.

Les sédiments déposés dans la retenue de barrage El Kansera contiennent dans l'ensemble, les mêmes teneurs en phosphore total. On constate une légère augmentation de ces teneurs au niveau des sédiments des stations B5 et B6. L'étude que nous avons effectuée des caractéristiques physico-chimiques des sédiments de la retenue a montré que ceux de ces deux stations (les plus profondes) ont la plus fine granulométrie. Cela pourrait expliquer cette augmentation des teneurs en phosphore total puisqu'il est bien établi que le phosphore se fixe préférentiellement sur les particules plus fines.

Lapplication du schéma de fractionnement chimique du phosphore aux sédiments de la retenue du barrage El Kansera, nous a permis de distinguer deux fractions minérales (phosphore lié au fer: $\mathrm{Fe}(\mathrm{OOH})-\mathrm{P}$ et phosphore lié au calcium : $\mathrm{CaCO}_{3}-\mathrm{P}$ ), ainsi que deux fractions organiques (phosphore organique soluble dans l'acide : POSA et phosphore organique résiduel : POR).

Les sommes des fractions extraites $\left\{\mathrm{Fe}(\mathrm{OOH})-\mathrm{P}+\mathrm{CaCO}_{3}-\mathrm{P}+\mathrm{POSA}+\mathrm{POR}\right\}$ des sédiments étudiés sont en général, inférieures aux teneurs en phosphore total. Ceci peut être dû à des pertes, inhérentes aux modes opératoires suivis lors des différentes extractions réalisées pour chaque échantillon. Toutefois, ces pertes, qui sont de l'ordre de $13 \%$, sont systématiquement reproductibles lors de toutes les extractions séquentielles effectuées ; ce qui diminue leur incidence sur la fiabilité des résultats.

D'un autre côté, les sédiments déposés dans la retenue (stations B1 à B6) et ceux déposés sur l'oued Beht (station OB), que ce soit en novembre 92 ou en janvier 94 , renferment des quantités assez comparables entre elles, des fractions du phosphore liées au fer et de celles liées au calcium. En effet, les teneurs des formes $\mathrm{Fe}(\mathrm{OOH})-\mathrm{P}$ et $\mathrm{CaCO}_{3}-\mathrm{P}$ varient respectivement entre 0,04 et $0,10 \mathrm{mg} \cdot \mathrm{g}^{-1}$ et 0,03 et $0,06 \mathrm{mg} \cdot \mathrm{g}^{-1}$. Cette faible variation est enregistrée aussi, mais dans une moindre mesure, dans le cas du phosphore organique résiduel. Les teneurs de cette forme varient entre 0,12 et $0,25 \mathrm{mg} \cdot \mathrm{g}^{-1}$ de sédiment sec.

Cependant, le phosphore organique soluble dans l'acide (POSA) présente une exception. Les sédiments prélevés lors de la période d'étiage renferment des teneurs en POSA qui sont stables dans l'espace $\left(0,03\right.$ à $\left.0,09 \mathrm{mg} \cdot \mathrm{g}^{-1}\right)$, mais beaucoup moins importantes que celles déterminées dans les sédiments déposés en période de crue $\left(0,24\right.$ à $\left.0,42 \mathrm{mg} \cdot \mathrm{g}^{-1}\right)$. Cette forme représente moins de $10,7 \%$ du phosphore total en période d'étiage, alors qu'en période de crue, elle représente plus de $25 \%$ voire $56 \%$ du phosphore total. Cette constatation pourrait s'expliquer par le fait que les matières organiques sont dégradées en période d'étiage; alors qu'en période de crue elles sont fraîches étant donné que les apports sont continus. D'un autre côté, les conditions anaérobies des couches hypolimniques et les faibles apports exogènes venant du bassin versant en périodes d'étiage peuvent favoriser la minéralisation du phosphore organique en phosphore minéral. 
Les sédiments recueillis au niveau de l'oued D'kor en novembre 92, qui ont la teneur la plus importante en phosphore total $\left(1,1 \mathrm{mg} \cdot \mathrm{g}^{-1}\right)$, renferment les teneurs les plus élevées en $\mathrm{Fe}(\mathrm{OOH})-\mathrm{P}\left(0,34 \mathrm{mg} \cdot \mathrm{g}^{-1}\right)$ et en $\mathrm{CaCO}_{3}-\mathrm{P}\left(0,11 \mathrm{mg} \cdot \mathrm{g}^{-1}\right)$. Cette observation peut être liée à la nature chimique des sédiments de cette station qui renferment des teneurs importantes en $\mathrm{CaCO}_{3}$ (23 à $45 \%$ ).

Les teneurs en phosphore lié au fer sont, dans l'ensemble, nettement supérieures aux teneurs en phosphore lié au calcium. Ceci est en accord avec les résultats trouvés par GOLTERMAN (1982) sur des sédiments ayant une forte teneur en $\mathrm{CaCO}_{3}$ (10 à $30 \%$ ). Par contre, FABRE et al. (1996) ont fait une observation contraire dans les sédiments de la rivière Garonne en France (en moyenne $0,092 \mathrm{mg} \cdot \mathrm{g}^{-1}$ pour le $\mathrm{Fe}(\mathrm{OOH})-\mathrm{P}$ et $0,124 \mathrm{mg} \cdot \mathrm{g}^{-1}$ pour le $\left.\mathrm{CaCO}_{3}-\mathrm{P}\right)$.

Par ailleurs, la figure 3 représente les résultats de l'analyse factorielle des correspondances sur le premier plan factoriel.

Les individus sur la figure 3 sont bien répartis selon la période de prélèvement. Les prélèvements effectués en janvier 1994 (hautes eaux) sont situés du côté positif de l'axe 1. Par contre, ceux effectués en novembre 1992 (basses eaux) sont situés du côté négatif de cet axe.

La période de hautes eaux (janvier 1994) est surtout caractérisée par la forme du phosphore organique soluble dans l'acide. Alors qu'en période de basses eaux (novembre 1992), ce sont les formes inorganiques qui sont les plus importantes. Cette différentiation pourrait être due aux dynamiques de transport des particules minérales et organiques en suspension qui sont différentes selon le niveau du plan d'eau et aussi, aux apports des matières organiques d'origine exo ou endogène.

Les stations $O D$ et $O B$ situées sur les deux oueds en amont du barrage (mises comme observations supplémentaires lors de l'analyse factorielle) sont en général assez loin des autres points, surtout pour OD en 1992 qui sort complètement du cadre. Cela est fort probablement lié au fait que durant cette période de basses eaux, l'Oued D'kor ne transporte pratiquement que des eaux usées de la ville de Khemisset, et ses apports en phosphore sont relativement importants par rapport à ceux de l'oued Beht.

\subsection{Relations entre les formes de phosphore}

La détermination des parts des fractions $\mathrm{Fe}(\mathrm{OOH})-\mathrm{P}$ et $\mathrm{CaCO}_{3}-\mathrm{P}$ est très importante, du fait que leur somme $\left(P_{\text {inorg }}\right)$ représente la fraction du phosphore biodisponible, pour certaines espèces de Scenedesmus, comme il a été montré par plusieurs auteurs (DE GRAAF BIERBRAUWER-WÜRTZ et GOLTERMAN, 1989 ; GROBLER et DAVIES, $1979 ; 1981)$. D'autres travaux ont montré que les formes restant après l'extraction de $\mathrm{Fe}(\mathrm{OOH})-\mathrm{P}$ et de $\mathrm{CaCO}_{3}-\mathrm{P}$, ne sont pas biodisponibles pour ces espèces. Cependant, le POSA peut devenir biodisponible après reminéralisation (assèchement des sédiments) (GOLTERMAN et DE GROOT, 1994).

Pour les sédiments de la retenue de barrage El Kansera les résultats de l'analyse factorielle des correspondances présentés par la figure 3 montrent que les variables $\mathrm{P}_{\text {bio }}$ (analysée comme variable complémentaire), $\mathrm{Fe}(\mathrm{OOH})-\mathrm{P}, \mathrm{CaCO}_{3}-\mathrm{P}$ et $P_{\text {inorg }}$ sont bien corrélées entre elles et sont reliées négativement avec le premier axe. Il en ressort que le phosphore biodisponible estimé par la méthode des bioessais se regroupe avec les formes $\mathrm{Fe}(\mathrm{OOH})-\mathrm{P}, \mathrm{CaCO}_{3}-\mathrm{P}$ et $\mathrm{P}_{\text {inorg. }}$. Ce résul- 


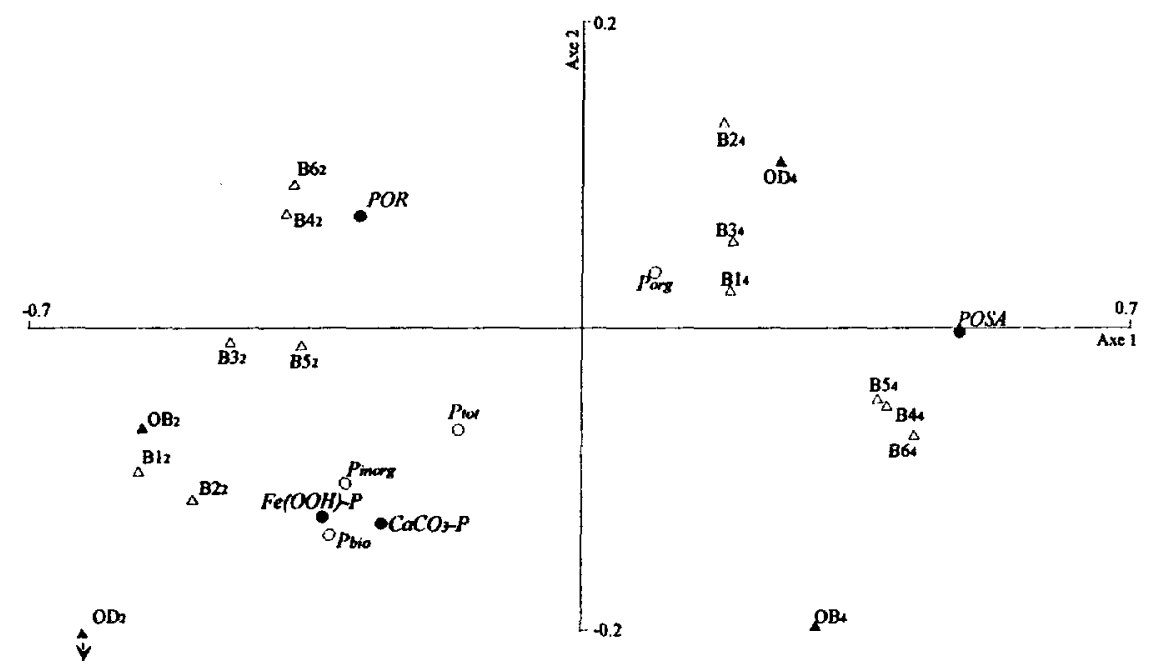

Figure 3 Résultats de l'analyse factorielle des correspondances : carte factorielle des stations et des variables sur le plan $F 1$ - F2.

$\mathrm{B} 12, \mathrm{~B} 22, \mathrm{~B} 32, \mathrm{~B} 42, \mathrm{~B} 52, \mathrm{~B} 62, \mathrm{OB} 2, \mathrm{OD} 2$, Stations prospectées en novembre 1992, B14, B24, B34, B44, B54, B64, OB4, OD4, Stations prospectées en janvier 1994.

Results of the factorial correspondence analysis: plots of sampled sites and phosphorus species in the $F 1$ - $F 2$ plane.

$B 12, B 22, B 32, B 42, B 52, B 62, O B 2, O D 2$, Stations sampled in November 1992, B14, B24, B34, B44, B54, B64, OB4, OD4, Stations sampled in January 1994.

tat est confirmé si nous consultons la matrice de corrélations (tabl. 2), puisque les coefficients de corrélations entre le $\mathrm{P}_{\text {bio }}$ et $\mathrm{Fe}(\mathrm{OOH})-\mathrm{P}, \mathrm{CaCO}_{3}-\mathrm{P}$ et $\mathrm{P}_{\text {inorg }}$ sont respectivement de 0,$820 ; 0,821$ et 0,827 .

Ceci est en accord avec les résultats trouvés récemment par FABRE et al. (1996) sur les sédiments de la rivière Garonne en France. Les coefficients de corrélation entre le $P_{\text {bio }}$ et les formes extraites par NTA $\{\mathrm{Fe}(\mathrm{OOH})-\mathrm{P}\}$ et par Na-EDTA $\left\{\mathrm{CaCO}_{3}-\mathrm{P}\right\}$ sont respectivement égaux à 0,88 et 0,77 .

Cependant, SIVADIER (1992), qui a trouvé de bonnes corrélations entre $P_{\text {bio }}$ et $\mathrm{Fe}(\mathrm{OOH})-\mathrm{P}(0,93)$ et $\mathrm{P}_{\text {inorg }}(0,94)$, a montré que le phosphore biodisponible pour Scenedesmus crassus se corrèle assez faiblement $(0,41)$ avec $\mathrm{CaCO}_{3}-\mathrm{P}$ extrait par $\mathrm{Na}$-EDTA dans les sédiments de la rivière Lot, en France. Ce résultat semble être lié aux caractéristiques physico-chimiques des sédiments analysés. En effet, les sédiments du Lot renferment des teneurs faibles en carbonates (entre 0 et $3,8 \%$ ) (SIVADIER, 1992); alors que dans les sédiments de la retenue de barrage El Kansera ces teneurs, d'après notre étude, sont plus importantes (entre 10 et $48 \%$ ).

Par ailleurs, la bonne corrélation trouvée entre la forme liée au fer et le phosphore biodisponible est en accord avec de nombreux travaux qui ont montré qu'une grande partie du phosphore lié au fer et du phosphore lié à l'aluminium sont biodisponibles quelle que soit la méthode d'extraction (DORICH et al., 1980 ; WILLIAMS et al., 1980). 
Tableau 2 Matrice de corrélations de Pearson, entre les différentes variables étudiées.

++ hautement significative : $R>0,623$.

+ significative : $0,497<R<0,623$.

- non significative : $R<0,497$.

Table 2 Pearson correlation matrix between the different variables studied.

++ highly significant $: R>0.623$.

+ significant : $0.497<R<0.623$.

- not significant : $R<0.497$.

\begin{tabular}{|lrrrrcccc|}
\hline & $\mathrm{Fe}(\mathbf{O O H})-\mathbf{P}$ & $\mathrm{CaCO}_{3}-\mathbf{P}$ & $\mathbf{P O S A}$ & $\mathrm{POR}$ & $\mathbf{P}_{\text {tot }}$ & $\mathbf{P}_{\text {inorg }}$ & $\mathbf{P}_{\text {org }}$ & $\mathbf{P}_{\text {bio }}$ \\
\hline $\mathrm{Fe}(\mathrm{OOH})-\mathrm{P}$ & 1,000 & \multicolumn{1}{c}{++} & - & - & + & ++ & - & ++ \\
$\mathrm{CaCO}_{3}-\mathrm{P}$ & 0,950 & 1,000 & - & - & ++ & ++ & - & ++ \\
$\mathrm{POSA}$ & $-0,320$ & $-0,232$ & 1,000 & - & - & - & ++ & - \\
$\mathrm{POR}$ & 0,253 & 0,291 & $-0,378$ & 1,000 & - & - & - & - \\
$\mathrm{P}_{\text {tot }}$ & 0,569 & 0,678 & $-0,065$ & 0,160 & 1,000 & + & - & + \\
$\mathrm{P}_{\text {inorg }}$ & 0,997 & 0,969 & $-0,303$ & 0,273 & 0,597 & 1,000 & - & ++ \\
$\mathrm{P}_{\text {org }}$ & $-0,271$ & $-0,166$ & 0,964 & $-0,118$ & $-0,021$ & $-0,246$ & 1,000 & - \\
$\mathrm{P}_{\text {bio }}$ & 0,820 & 0,821 & $-0,394$ & 0,408 & 0,608 & 0,827 & $-0,306$ & 1,000 \\
\hline
\end{tabular}

D'un autre côté, les formes $P_{\text {bio }}, \mathrm{Fe}(\mathrm{OOH})-\mathrm{P}, \mathrm{CaCO}_{3}-\mathrm{P}$ et $\mathrm{P}_{\text {inorg }}$ sont notablement indépendantes des formes POSA et $P_{\text {org }}$ (fig. 3). Les coefficients de corrélation sont en effet relativement bas $(R<0,41)$. Quant au phosphore organique résiduel (POR), il semblerait non lié à aucune variable (tabl. 2). Il en résulte donc que les formes organiques sont non biodisponible pour l'espèce Scenedesmus crassus.

Le phosphore total $P_{\text {tot }}$ occupe, sur la figure 3 , une position centrale par rapport aux autres variables, justifiant ainsi le fait qu'il englobe toutes les formes du phosphore. En revanche, il corrèle significativement avec la forme biodisponible ; le coefficient de corrélation entre $P_{\text {tot }}$ et $P_{\text {bio }}$ est de 0,61 . Ceci est en accord avec les résultats de WILLIAMS (1980), qui ont montré que la quantité de phosphore sédimentaire utilisable par les algues est relativement proportionnelle à la concentration du phosphore total lié aux sédiments.

\section{4- CONCLUSION}

L'application du schéma des extractions séquentielles chimiques du phosphore, lié aux sédiments de la retenue de barrage El Kansera, nous a permis d'évaluer les proportions de quatre formes différentes de phosphore: forme liée au fer $\{\mathrm{Fe}(\mathrm{OOH})-\mathrm{P}\}$, forme liée au calcium $\left\{\mathrm{CaCO}_{3}-\mathrm{P}\right\}$, forme organique soluble dans l'acide $\{P O S A\}$ et forme organique résiduelle $\{P O R\}$. Le phosphore biodisponible pour l'espèce Scenedesmus crassus, qui représente moins de $32 \%$ du phosphore total des sédiments étudiés, a été par ailleurs évalué par les tests des bioessais.

II en ressort que dans la retenue du barrage EI Kansera, la répartition des formes du phosphore dans les sédiments semble être influencée par les caractéristiques physico-chimiques des sédiments étudiés, qui dépendent de la période et 
du lieu de prélèvement. La première période (basses eaux) est caractérisée par la prédominance des formes inorganiques de phosphore; alors qu'en période de hautes eaux, le phosphore organique soluble dans l'acide est le plus important.

Les formes inorganiques $\left\{\mathrm{Fe}(\mathrm{OOH})-\mathrm{P}\right.$ et $\left.\mathrm{CaCO}_{3}-\mathrm{P}\right\}$ sont fortement liées au phosphore biodisponible, déterminé par la méthode de bioessais. En effet, le coefficient de corrélation dépasse 0,82 . II a été montré en outre, que le phosphore biodisponible n'est pas significativement lié aux formes organiques \{POSA et POR] dans les sédiments de la retenue de barrage EI Kansera.

Nous sommes conscients que le présent jeu de données est insuffisant pour nous permettre de conclure avec certitude. Cependant, on peut affirmer que lapproche utilisée ici, couplant des biotests avec des analyses de spéciation du phosphore, permet de dégager des tendances intéressantes et ouvre des perspectives pour des travaux futurs.

En particulier, on a vu que la biodisponibilité du phosphore lié au calcium dans les sédiments est contesté par certains auteurs. Il semble également raisonnable de penser que la biodisponibilité des formes inorganiques et en particulier celles liées au calcium dépend de la nature physico-chimique des sédiments.

Il paraît judicieux par conséquent de poursuivre nos recherches, en vérifiant si les tendances relevées ici se confirment, notamment en prenant en considération le facteur temps et la recherche des paramètres physico-chimiques précis dont dépend la biodisponibilité du phosphore.

\section{RÉFÉRENCES BIBLIOGRAPHIQUES}

AFDALI, M. (1993). Contribution à l'étude du comportement du phosphore dans le lac réservoir Hassan $1^{\text {er }}$ (Maroc) : Spéciation, dynamique et biodisponibilité du phosphore particulaire des sédiments. Thèse de D.E.S. de $3^{\ominus}$ cycle, Faculté des Sciences Semlalia, Marrakech.

BENZECRI, J.-P. (1973). L'analyse des données. II l'analyse des correspondances. Dunod ed., Paris, 619 p.

BONZONGO, J. (1990). Phosphore et sédiments : méthodes d'analyse - stockage - relargage - biodisponibilité. Thèse d'Université, Université de Rennes I.

BONZONGO, J., BERTRU, G., MARTIN, G. (1989). Les méthodes de spéciation du phosphore dans les sédiments : Critiques et propositions pour l'évaluation des fractions minérales et organiques. Arch. Hydrobiol., 116, 1, 61-69.
BOSTROM, B., PERSSON, G., BROBERG, B. (1988). Bloavailability of different phosphorus forms in freshwater systems. Hydrobiologia, 170, 133-155.

DE GRAAF BIERBRAUWER-WURTZ, I.M., GOLTERMAN, H.L. (1989). Fosfaat fracties in de bodem van een aantal Nederlandse meren. $\mathrm{H}_{2} \mathrm{O}, 22,411-414$.

DE GROOT, C.J., GOLTERMAN, H.L. (1990). Sequential fractionation of sediment phosphate. Hydrobiologia, 192, 143-148.

DE GROOT, C.J. (1992). Flux et transformations du phosphate et de l'azote dans les zones humides: la Camargue (Delta du Rhône). Thèse d'Université, Université Claude Bernard, Lyon I.

DERRAZ, M. (1995). Etude de l'eutrophisation de la retenue du barrage EI Kansera (Maroc). Caractéristiques physico-chimi- 
ques, biodisponibilité du phosphate sédimentaire, ecophysiologie de Microcystis et relation des blooms avec les paramètres environnementaux. Thèse de Doctorat ès-Sciences. Université Moulay Ismaïl, Meknès.

DERRAZ, M., DAUTA, A., CAPBLANCQ, J., ABBASSI, M. (1995). Influence de la lumière et de la température sur les taux de croissance et de photosynthèse de Scenedesmus crassus Chodat, isolée de la retenue eutrophe El Kansera (Maroc). Annis. Limnol., 31, 1, 65-74.

DILLON, P.J., RIGLER, F.H. (1974). The phosphorus - chlorophyll relationship in lakes. Limnol. Oceanogr., 19, 767-773.

DORICH, R.A., NELSON, D.W., SOMMER, L.E. (1980). Algal availability of sediment phosphorus in drainage water of the black creek watershed. J. Environ. Qual., 8, 577-563.

FABRE, A., QOTBI, A., DAUTA, A., BALDY, V. (1996). Relation between algal available phosphate in the sediments of River Garonne and chemically-determined phosphate fractions. Hydrobiologia, 335 , 43-48.

GOLTERMAN, H.L., BOOMAN, A. (1988). Sequential extraction of iron-phosphate and calcium-phosphate from sediments by chelating agents. Verh. Internat. Verein. Limnol., 23, 904-909.

GOLTERMAN, H.L., DE GROOT, C.J. (1994). Nouvelles connaissances des formes du phosphate : conséquences sur le cycle du phosphate dans les sédiments des eaux douces peu profondes. Annls Limnol., 30, 3, 221-232.

GOLTERMAN, H.L. (1982). Differential extraction of sediment phosphates with NTA solutions. Hydrobiologia, 92, 683-687.

GOLTERMAN, H.L. (1988). Reflexions on fractionation and bioavailability of sediment bound phosphate. Arch. Hydrobiol. Beih., 30, 1-4.

GROBLER, D.C., DAVIES, E. (1979). The availability of sediment phosphate to algae. Water $S A, 5,114-123$.

GROBLER, D.C., DAVIES, E. (1981). Sediment as source of phosphate ; A study of 38 impoundments. Water SA, 7, 54-60.

HANNA, M., DAUTA, A. (1983). Bioessays a comparative study of three parametrs related to phosphorus bioavailability (yield, growth rate and interacellular concentration of phosphorus). Ann/s. Limnol., 19, 1, 59-66

HIELTJES, A.H.M., LIJKLEMA, L. (1980). Fractionation of inorganic phosphates in calcareous sediments. J. Environ. Qual., 9, 405-407.

LAFFORGUE, M. (1990). Modélisation du fonctionnement d'un écosystème lacustre : le lac d'Aydat. Thèse de Doctorat de l'Ecole des Mines, Paris.

LAHLOU, A. (1987). Etude actualisée de l'envasement des barrages au Maroc. Sciences de l'Eau, 6, 3, 335-356.

MOTOMIZU, S., WAKIMOTO, T., TOEI, $K$. (1983). Spectrophotometric determination of phosphate in river waters with molybdate and malachite green. Analyst, March, 108, 361-367.

MURPHY, J., RILEY, J.P. (1962). A modified single solution method for determination of phosphate in natural waters. Anal. Chim. Acta, 27, 31-36.

OLILA, O.G., REDDY, K.R., HARRIS, W.G. (1995). Forms and distribution of inorganic phosphorus in sediments of two shallow eutrophic lakes in Florida. Hydrobiologia, 302, 147-161.

PSENNER, R., PUCSKO, R. (1988). Phosphorus fractionation: Advantages and limits of the method for study of sediment $P$ origins and interactions. Arch. Hydrobiol. Beith Ergebn Limnol, 30, 43- 59.

RECKHOW, K.H., CHAPRA, S.C. (1983). Engineering approaches for lakes management. Volume 1: Data analysis and empirical modeling. Butterworth Publishers, $340 \mathrm{p}$.

SIVADIER, F. (1992). Etude comparée de deux méthodes d'évaluation de la biodisponibilité du phosphore sédimentaire: bioessais et extraction séquentielle par des agents chelatants. D.E.A d'écologie, Université Paul Sabatier, Toulouse III, $46 \mathrm{p}$.

VOLLENWEIDER, R.A. (1976). Advances in defining critical loading levels for phosphorus in lake eutrophication. Mem. Ist. Ital. Idrobiol., 33, 53-83.

WILLIAMS, J.D.H., SHEAR, H., THOMAS, R.L. (1980). Availability to Scenedesmus quadricauda of different forms of phosphorus in sedimentary materials from the Great Lakes. Limnol. Oceanogr., 25, 1-11. 